\title{
Enhanced electron screening in nuclear reactions and radioactive decays
}

\author{
K. Czerski ${ }^{1,2}{ }^{\star}$, P. Heide ${ }^{2}$, A. Huke ${ }^{2}$, L. Martin ${ }^{2}$, G. Ruprecht ${ }^{3}$ \\ ${ }^{1}$ Institute of Physics, University of Szczecin \\ ul. Wielkopolska 15, 70-451 Szczecin, Poland \\ ${ }^{2}$ Institut für Optik und Atomare Physik, Technische Universität Berlin \\ Hardenbergstr. 36, 10623 Berlin, Germany \\ ${ }^{3}$ TRIUMF National Laboratory \\ 4004 Wesbroock Mall, Vancouver, BC, V6T 2A3, Canada \\ E-mail: czerski@kalium.physik.tu-berlin.de
}

\begin{abstract}
In recent years, an enhanced electron screening in metallic environments has been demonstrated by many groups in experimental investigations of low-energy nuclear reactions. Similarly, first radioactive decay experiments in metallic materials have been performed to possibly observe an alteration of the decay constant due to electron screening. Both kinds of experiments are of fundamental importance for nuclear astrophysics since the metallic quasi-free electrons represent a model for dense astrophysical plasmas and thus the corresponding theories can be experimentally verified. Here, the experimental results determined for $\mathrm{d}+\mathrm{d}$ fusion reactions will be applied to propose a scaling procedure for electron screening energies in different metallic materials. The results will be compared with the experimental values obtained for reactions with heavier nuclei and some predictions for radioactive decay experiments will be presented. Additionally, the temperature dependence of the screening energy will be discussed.
\end{abstract}

International Symposium on Nuclear Astrophysics - Nuclei in the Cosmos - IX

CERN, Geneva, Switzerland

25-30 June, 2006

\footnotetext{
* Speaker
} 


\section{Introduction}

Already first observations of the enhanced electron screening effect in the $d+d$ fusion reactions in metallic environments have been motivated by its importance for dense astrophysical plasmas [1]. From the theoretical point of view the deuterized metals can be treated as a strongly coupled plasma and therefore provide a unique possibility for experimental studies of nuclear pycnoreactions under terrestrial conditions. The theoretical calculations [2] based on the self-consistent dielectric function theory can qualitatively explain the target material dependence of the experimentally determined screening energies, however, the absolute theoretical values are underestimated by a factor of two. The reason for this discrepancy is still unknown. Large screening energies in metallic environments have been also demonstrated by other groups [3,4]. Some differences between experimental results obtained by different groups probably result from a target surface contamination or an inhomogeneous distribution of deuterons within the target [5]. Generally, the experimental screening energies reach about $300 \mathrm{eV}$ for heavier metals and are much smaller (below $100 \mathrm{eV}$ ) for insulating materials.

The results obtained for $d+d$ reactions can be verified in reactions with heavier nuclei. Recently, large screening energies have been observed for the ${ }^{6} \mathrm{Li}+\mathrm{d}$ system [6] and confirmed by the LUNA collaboration [7]. In the present work, we would like to provide a scaling procedure allowing to predict the screening energies for heavier reaction systems. On the other hand, the same procedure can be applied for studies of radioactive decays taking place in different environments. Large differences between the screening energies for insulating and metallic environments enable to anticipate a corresponding half-life alteration for alpha and beta decays. Additionally, a possible temperature dependence of the screening energy as proposed in $[8,9]$ will be discussed.

\section{Electron screening in nuclear reactions with heavy nuclei}

The electrostatic potential energy between reacting nuclei of charges $Z_{1}$ and $Z_{2}$ in a metallic medium can be described in analogy to dense astrophysical plasmas within the selfconsistent dielectric function theory [5]:

$$
V(r)=\frac{Z_{1} Z_{2} e^{2}}{r} \Phi(r)=\frac{Z_{1} Z_{2}}{(2 \pi)^{3}} \int \frac{4 \pi e \varphi_{1}(q) e \varphi_{2}(q)}{\varepsilon_{v}(q) \varepsilon_{c}(q) q^{2}} \exp (i \vec{q} \vec{r}) d^{3} q \approx \frac{Z_{1} Z_{2} e^{2}}{r}-U_{e}
$$

The wave-number dependent dielectric functions $\varepsilon_{v}$ and $\varepsilon_{c}$ describe polarization of valence and core electrons of host atoms induced by a charged impurity. $\Phi(r)$ and $\varphi_{i}(q)$ functions are the screening function and electronic charge-formfactors of reacting nuclei, respectively (for details see [2]). At small distances (applicable for nuclear reactions and decays) the potential energy can be approximated using the energy independent screening energy $U_{e}$. From Eq. 1 it can be found that the screening energy scales with the product of the charges of the involved 
nuclei.

The results obtained for the $\mathrm{d}+\mathrm{d}$ reactions taking place in different metallic targets indicate that the electron screening energies moderately increase with the atomic number of host atoms [5] reaching for heavier nuclei the value of about $300 \mathrm{eV}$. This target material dependence agrees with the theoretical expectations and mainly results from an increasing cohesion energy difference between two reacting deuterons and the compound nucleus ${ }^{4} \mathrm{He}$ embedded in the crystal lattice of heavier metals (so-called cohesion screening). On the other hand, the experimental screening energies are larger by a factor of about 2 compared to the theoretical values. Since the experimental screening energies obtained for insulating materials are much smaller $(<100 \mathrm{eV})[4,5]$ and taking into account that the screening contributions resulting from polarization of bound host electrons and cohesion should be similar for both metallic and insulating targets, we can conclude that the enhanced screening effect arises from conducting electrons. In the Thomas-Fermi model this contribution is given by $U_{e, f}(\mathrm{TF})$ $=Z_{1} Z_{2} \mathrm{e}^{2}\left[4 /\left(\pi a_{0}\right)\right]^{1 / 2}\left(3 \pi^{2} n\right)^{1 / 6}$ where $n$ and $a_{0}$ are the valence electron density and the Bohr radius, respectively. Using the electron-gas parameter $r_{s}=[3 /(4 \pi n)]^{1 / 3} / a_{0}$ we obtain $U_{e, f}(\mathrm{TF})$ $=2 Z_{1} Z_{2} \mathrm{e}^{2}\left[9 /\left(4 \pi^{2}\right)\right]^{1 / 6} r_{s}^{-1 / 2}=Z_{1} Z_{2} 42.5 \mathrm{eV} / r_{s}^{1 / 2}$. The same scaling can be applied for the experimental values determined for $\mathrm{Li}, \mathrm{Al}, \mathrm{Zr}$, Pd and Ta targets [5]. In order to estimate the experimental contribution of free electrons the experimental screening energies have to be reduced by contributions arising from bound electrons and the cohesion screening. Finally, we get $U_{e, f}=Z_{1} Z_{2}(250 \pm 20) \mathrm{eV} / r_{s}^{1 / 2}$, which can be used for an estimation of the free electron contribution in metallic environment to the screening energy in reactions between nuclei with charges $Z_{1}$ and $Z_{2}$. Different to the $\mathrm{d}+\mathrm{d}$ reactions, the contribution coming from electrons bound by heavier reacting nuclei is much larger and should be included separately. This can be calculated as the gain in electron binding energies between distant atoms and the final united atom. Similar results are to obtain using the Thomas-Fermi model leading to $U_{e, b}(\mathrm{TF})=1.13$ $Z_{1} Z_{2} \mathrm{e}^{2}\left(\mathrm{Z}_{1}^{1 / 2}+\mathrm{Z}_{2}^{1 / 2}\right)^{2 / 3} / a_{0}$ [10]. The total screening energy is the sum of the free electron and bound electron contributions.

The first experimental determination of the screening energy in metallic environments for heavier reaction systems $\left({ }^{6} \mathrm{Li}+\mathrm{d}\right)$ was performed by Kasagi et al. [6] and confirmed later by the LUNA collaboration [7] . In Tab.1 the experimental results for the ${ }^{6} \mathrm{Li}+\mathrm{d},{ }^{6} \mathrm{Li}+\mathrm{p}$ and ${ }^{7} \mathrm{Li}+\mathrm{p}$ systems in the insulating $(\mathrm{LiF})$ and metallic ( $\mathrm{Li}$ and $\mathrm{PdLi}_{\mathrm{x}}$ alloy) environments are presented and compared to the theoretical values evaluated according to the scaling given above. The screening energy for bound electrons calculated as the gain in the electron binding energies amounts to $190 \mathrm{eV}$ and agrees with the Thomas-Fermi estimation of $180 \mathrm{eV}$ as well as with the

Tab.1 Screening energies for ${ }^{6} \mathrm{Li}+\mathrm{d},{ }^{6} \mathrm{Li}+\mathrm{p}$ and ${ }^{7} \mathrm{Li}+\mathrm{p}$ systems

\begin{tabular}{|l|c|c|c|c|}
\hline \multirow{2}{*}{$\begin{array}{c}\text { host } \\
\text { material }\end{array}$} & \multicolumn{4}{c|}{ screening energy (eV) } \\
\cline { 2 - 5 } & bound & free & theo (bound+free) & exp \\
\hline insulator & 190 & - & 190 & $190^{7,11}$ \\
\hline Lithium & 190 & $3 \cdot 140$ & 610 & $1200^{7}$ \\
\hline PdLi $_{\mathrm{x}}$ & 190 & $3 \cdot 220$ & 850 & $1500^{6}-3500^{7}$ \\
\hline
\end{tabular}




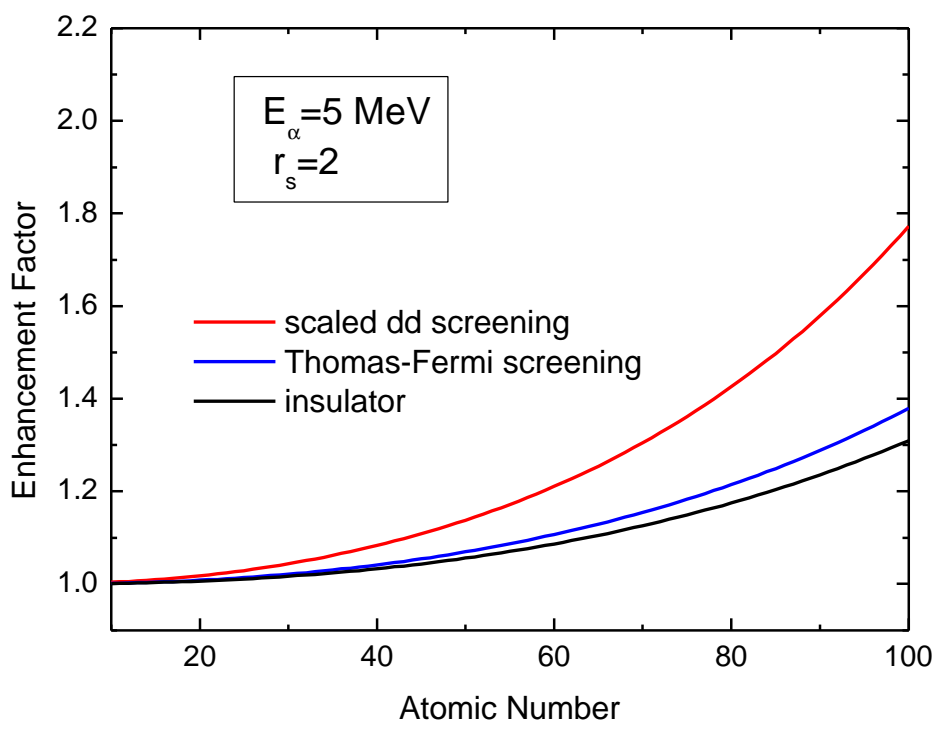

Fig. 1 Enhancement factors for alpha-decay $\left(E_{\alpha}=5 \mathrm{MeV}\right)$ in metallic $\left(r_{\mathrm{s}}=2\right)$ and insulating environments

experimental results. The free electron contribution to the screening energy is different for metallic Li and Pd environments because of different electron densities. A comparison between theoretical and experimental screening energies is presented in Tab.1. The errors of the experimental screening energies are relatively large mainly due to systematic uncertainties. Nevertheless, both theoretical and experimental data confirm a strong target material dependence and much larger screening energies than for the $\mathrm{d}+\mathrm{d}$ reactions.

\section{Electron screening in radioactive decays}

In radioactive decays, where the emitted particle interacts with the recoil nucleus electrostatically, the electron screening effect plays an important role. In alpha decays alpha particles have to tunnel the Coulomb barrier in opposite direction compared to low energy nuclear reactions. Thus, the probability for alpha-decay depends on the electronic structure of both the decaying atom and the host medium. Assuming that the change of the Coulomb barrier due to surrounding electrons can be described by a screening energy $U_{e}$ and the binding energy of the decaying nucleus remains unaffected we obtain an expression for an increase of the decay probability compared to the bare nucleus case as follows:

$$
f=\frac{P\left(E+U_{e}\right)}{P(E)}=\frac{\sqrt{\frac{E_{G}}{E+U_{e}}} \exp \left(-\sqrt{\frac{E_{G}}{E+U_{e}}}\right)}{\sqrt{\frac{E_{G}}{E}} \exp \left(-\sqrt{\frac{E_{G}}{E}}\right)} \approx \exp \left(\frac{U_{e}}{2 E} \sqrt{\frac{E_{G}}{E}}\right)
$$




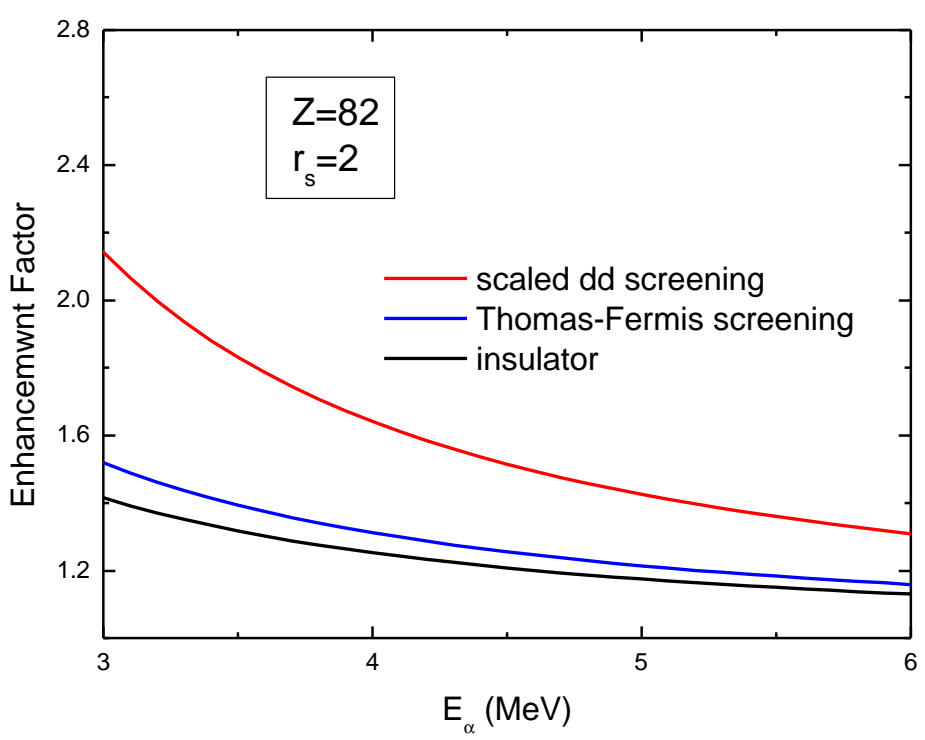

Fig. 2 Enhancement factors for alpha decay $(Z=82 \mathrm{MeV})$ in metallic $\left(r_{\mathrm{s}}=2\right)$ and insulating environments

Here, the enhancement factor $f$ is the ratio of the corresponding penetration factors for screened and bare nuclei. The approximation in Eq. (2) can be made when the alpha decay energy E is much larger than the screening energy $U_{e}$. The Gamow energy is given by $E_{G}=\left(\frac{4 \pi Z e^{2}}{\hbar c}\right)^{2} \frac{\mu}{2}$ where $\mu$ denotes the reduced mass. In the case of metallic environments, the screening energy $U_{e}$ consists of two components: one results from bound electrons of the decaying atom and the other from quasi-free conduction electrons of the metal. Similar to nuclear reactions with heavy nuclei the cohesion screening contribution as well as polarization of bound electrons of the host atoms can be neglected. For insulating environments we only have the bound electron contribution. Since the binding energies of inner atomic electrons depend only very slightly on the crystal lattice structure, we assume that this contribution has the same form for insulating and metallic materials and can be described by the Thomas-Fermi model. The enhancement factor for the alpha decay with $E_{\alpha}=5 \mathrm{MeV}$ calculated for metallic $\left(r_{s}=2\right)$ and insulating environments is presented in Fig.1: The higher atomic number of the decaying nucleus the larger difference between half-lives in insulators and metals. For ${ }^{238} \mathrm{U}$ and $E_{\alpha}=5 \mathrm{MeV}$ we get $f=1.25$ for insulating and $f=1.31$ or 1.61 for metallic $\left(r_{s}=2\right)$ environments using Thomas-Fermi model or $d+d$ scaling. In Fig. 2 the dependence of the enhancement factor on the alpha-decay energy is presented.

The energy spectrum of emitted positrons (+) and electrons (-) in allowed nuclear betadecays can be written as follows: 


$$
d N^{ \pm}=C|\langle M\rangle|^{2} F^{ \pm}(Z, W) p W\left(W_{0}-W\right)^{2} d W
$$

where $\mathrm{C},|\langle M\rangle|$ and $F^{ \pm}$are a constant factor, the transition matrix and the Fermi function for positrons or electrons, respectively. $W$ and $p$ denote the total energy and the momentum of beta particles, $W_{0}$ corresponds to the maximum energy. The Fermi function describes Coulomb interaction between the beta particles and the decaying nucleus and changes the energy spectrum as well as the total decay probability such that $\beta^{-}$decay is more probable than $\beta^{+}$. When the Coulomb barrier is shielded by surrounding electrons the total $\beta^{-}$decay probability decreases (half-life increases) and the $\beta^{+}$decay probability increases (half-life decreases). Numerically, the electron screening can be taken into account by replacing the total electron energy $W$ in the Fermi function into $W^{\prime}=W+U_{e}$ as follows [12]:

$$
F_{s c r}^{ \pm}(Z, W)=F^{ \pm}\left(Z, W^{\prime}\right) \frac{p^{\prime} W^{\prime}}{p W}
$$

The screening energy $U_{e}$ can be calculated similar to alpha decay. The influence of the screening effect on beta decay rates is not so strong as for alpha decay. In Fig.3 the enhancement factor (ratio between the decay probability for screened and bare nucleus) for $\mathrm{B}^{+}$ decay of $W_{0}=1.511 \mathrm{MeV}\left(E_{\beta}=1 \mathrm{MeV}\right)$ is presented. Although the enhancement factors are relatively small they should be determinable experimentally.

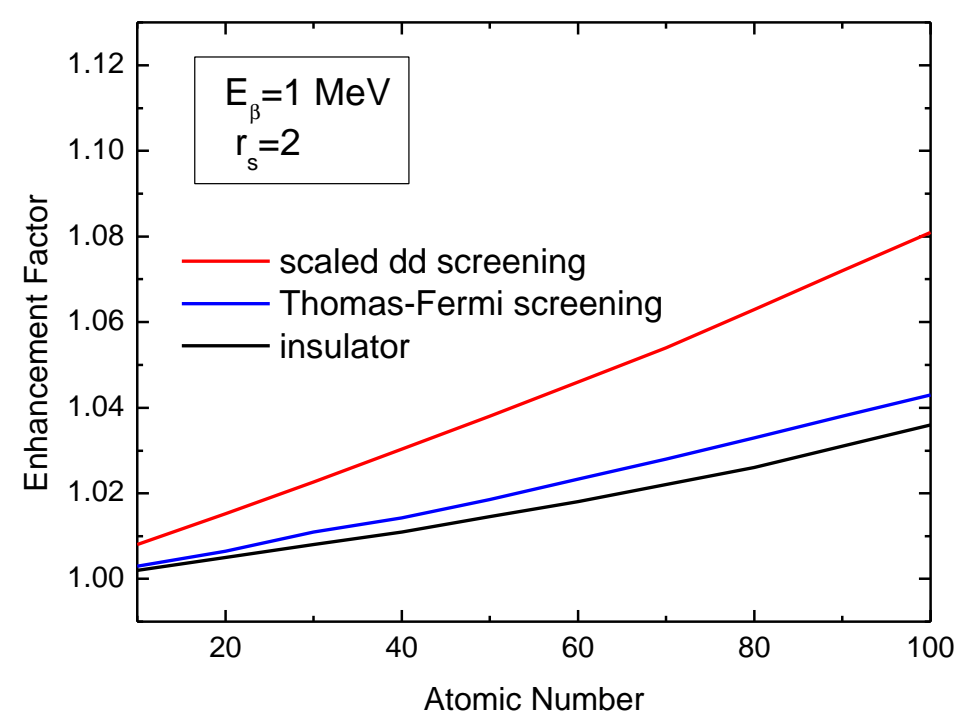

Fig. 3 Enhancement factors for $\beta^{+}$decay $\left(E_{\beta}=1 \mathrm{MeV}\right)$ in metallic $\left(r_{\mathrm{s}}=2\right)$ and insulating environments 


\section{Temperature dependence of the screening}

The suggestion [9] that the enhanced electron screening in metallic environments could be described by the Debye-Hückel theory would imply a strong temperature dependence of the screening energy $\sim 1 / \sqrt{T}$. As already pointed out in [5] the Debye-Hückel screening (so-called weak screening) is applicable only for high temperatures or equivalently for high projectile energies. At lower temperatures corresponding in metallic environments to velocities below the Fermi velocity the electron screening reaches the strong-screening limit. The screening energy dependence on the projectile energy (or on temperature according to the equation $\mu v^{2} / 2=3 / 2 k T$ ) calculated for the $\mathrm{d}+\mathrm{d}$ fusion reactions in $\mathrm{Al}$ is presented in Fig.4 . The transition from the weak screening to the strong screening takes place at the Fermi energy of $20 \mathrm{keV}$ for $\mathrm{Al}$ and correspondingly at the Fermi temperature of about $10^{5} \mathrm{~K}$. For lower energies or temperatures the screening energy changes only very slightly (blue curve).

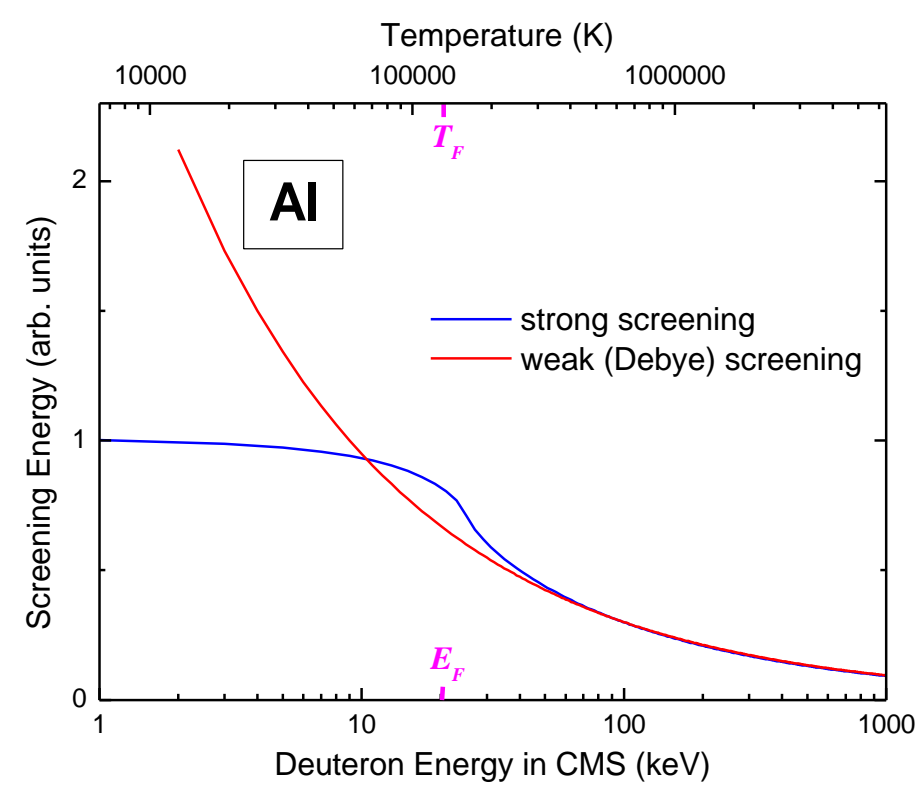

Fig.4 Screening energy dependence on the temperature resp. projectile energy

\section{Conclusions and discussion}

Large experimental values of screening energies obtained for $d+d$ fusion reactions provide interesting consequences for nuclear reactions with heavier nuclei where the screening enhancement effect should be stronger. Based on dielectric function theory some theoretical estimation can be made for which a scaling of the screening energy with the atomic number has been proposed. Differences in the screening energies for insulating and metallic environments lead to a prediction of alteration of half-lives for radioactive decays in various media. Especially for alpha decays the shortening of half-life due to the electron screening should be easy to prove. However, an additional energy shift of the alpha decaying state due to the electron 
screening, which has not been included in the present analysis, can reduce the predicted enhancement. The screening effect in beta decays, although weaker, could also be experimentally verified. Thus, future experimental studies of the screening effect in radioactive decays offer a new possibility to clarify discrepancies between theoretical and experimental screening energies observed in low-energy nuclear reactions. An independent confirmation of the observed enhanced screening effect in metallic environments should have far-reaching consequences for astrophysical plasmas as well as for some technical applications, for instance for the fusion-reactor technique. The Debye temperature dependence of the screening energy proposed in [8,9] is not applicable for metals. However, some small effects due to changes of electronic band structure might be expected (see also the experimental investigation of $\beta^{+}$decay of $\left.{ }^{22} \mathrm{Na}[13]\right)$.

\section{References}

[1] K. Czerski, A. Huke, P. Heide, M. Hoeft, G. Ruprecht, Nuclei in the Cosmos V, Proceedings of the International Symposium on Nuclear Astrophysics, July 6-11, 1998 Volos, Greece, edited by N. Prantzos and S. Harissopulos (Editions Frontières) p.152

K. Czerski, A. Huke, A. Biller, P. Heide, M. Hoeft, G. Ruprecht, Europhys. Lett. 54 (2001) 449

[2] K. Czerski, A. Huke, P. Heide, G. Ruprecht, Europhys. Lett. 68 (2004) 363

[3] J. Kasagi et al., J. Phys. Soc. Jpn. 71 (2002) 2281

[4] F. Raiola et al., Eur. Phys. J. A 13 (2002) 377

[5] K. Czerski, A. Huke, P. Heide, G. Ruprecht, Eur. Phys. J. A 27 (2006) 83

[6] J. Kasagi et al., J. Phys. Soc. Jpn. 73 (2004) 608

[7] J. Cruz et al., Phys. Lett. B 624 (2005) 181

[8] F. Raiola et al., J. Phys. G 31 (2005) 1141

[9] B. Limata et al., Eur. Phys. J. A 28 (2006) 251

[10] J.F. Ziegler, J.P. Biersack, U. Littmark, The Stopping and Ranges of Ions in Solids, Pergammon Press, New York, 1985

[11] G. Ruprecht, K. Czerski, D. Bemmerer, M. Hoeft, P. Heide, Phys. Rev. C 70 (2004) 025803

[12] L. Durand, III, Phys. Rev. 135 (1964) B310

[13] G. Ruprecht et al., this conference. 